\title{
Bayesian acoustic prediction assimilating oceanographic and acoustically inverted data
}

\author{
Nelson E. Martins and Sérgio M. Jesus \\ Institute for Systems and Robotics, University of Algarve, Campus de Gambelas, \\ PT-8005-139 Faro, Portugal
}

\begin{abstract}
The prediction of the transmission loss evolution on a day to week frame, in a given oceanic area, is an important issue in modeling the sonar performance. It relies primarily on acoustic propagation models, which convert water column and geometric/geoacoustic parameters to 'instantaneous' acoustic field estimates. In practice, to model the acoustic field, even the most accurate acoustic models have to be fed with simplified environmental descriptions, due to computational issues and to a limited knowledge of the environment. This is a limitation, for example, in acoustic inversion methods, in which, by maximizing the proximity between measured and modeled acoustic signals, the estimated environmental parameters are deviated from reality, forming what is normally called an 'acoustically equivalent environment'. This problem arises also in standard acoustic prediction, in which, the oceanographic forecasts and bottom data (typically from archives) are fed directly to an acoustic model. The claim in the present work is that, by converting the oceanographic prediction and the bottom properties to 'acoustically equivalent' counterparts, the acoustic prediction can be obtained in an optimal way, adapted to the environmental model at hand. Here, acoustic prediction is formulated as a Bayesian estimation problem, in which, the observables are oceanographic forecasts, a set of known bottom parameters, a set of acoustic data, and a set of water column data. The predictive posterior PDF of the future acoustic signal is written as a function of elementary PDF functions relating these observables and 'acoustically equivalent' environmental parameters. The latter are obtained by inversion of acoustic data. The concept is tested on simulated data based on water column measurements and forecasts for the MREA'03 sea trial.
\end{abstract}

Key words: acoustic prediction, oceanographic forecast, acoustic inversion, rapid environmental assessment

PACS: 43.30.+m 


\section{Introduction}

The problem of estimating the acoustic field in a given oceanic area (transect, volume, etc.) at a future time has triggered research in both oceanography and underwater acoustics areas[14,18]. Since acoustic propagation is strongly dependent on the space-time sound speed field, the oceanographic community has developed sophisticated oceanographic observation and prediction tools[14,18]. Essentially, these tools combine dynamic models based on the primitive equations, statistical models of the inter-correlation between oceanographic quantities, and data models, giving estimates of e.g. the tides, and the temperature, salinity and currents fields evolution[1,18]. By their side, the underwater acoustics community has developed sophisticated acoustic propagation models based on e.g. normal mode theory, ray theory, or the parabolic equation, able to give accurate predictions of the acoustic field[5]. These models compute the field as a function of environmental quantities, solving an acoustic modeling forward problem.

With the purpose of estimating the acoustic field at future time, and by linearization of water column descriptors on short time periods, a rough statistical estimator of the acoustic field could be based on acoustic correlation models in space-time, which would drive MMSE estimators, Kalman filters, etc. However, the application of such purely statistical predictors is unfeasible due to the highly nonlinear dependence of acoustic signals on environmental conditions. Thus, it is a common practice to run a given oceanographic model calibrated for the oceanic area of interest, and then to use the obtained sound speed forecasts and geometric/geoacoustic archival data as input to an acoustic propagation model[8-13,16-18]. For example, in [8], the uncertainty of the acoustic prediction is illustrated as a function of the oceanographic prediction uncertainty, via Monte Carlo forward simulations of the acoustic field. A more robust prediction system, including feedback, is outlined in $[10,12,18]$, with both oceanographic and acoustic prediction capabilities. Here, both oceanographic, acoustic data and models are merged to minimize an interdisciplinary cost function. Acoustic prediction has been treated so far as one product of coupled ocean-acoustics research, where the error of the predicted acoustics is dependent on the water column forecast error and the geometric/geoacoustic parameters accuracy. The latter can be weak, due to sparsity of bottom data or, for example, to the merely indicative character of the information found on nautical charts or historical databases. At the end, it is generally claimed that a decrease in the predicted acoustic signal error is attained only with a decrease in the environmental information error.

An important issue in acoustic prediction is that the subspace spanned by the acoustic signal is dependent on acoustic modeling constraints. These constraints are essentially threefold. First, computational issues limit the detail of 
the environmental description (of e.g. bathymetry and range-dependent sound speed). Second, the end-user environmental knowledge is often incomplete for the acoustic grid of interest (e.g. bottom properties and number of layers). Third, physical inaccuracies may take place due to the numeric approximations applied in solving the acoustic wave equation. From the environmental viewpoint, this implies that, for a given acoustic data set and the corresponding real environment, the simulated acoustics closest to the acoustic data has to be parameterized by an environment slightly shifted from the real environment and here designated as the 'acoustically equivalent environment'. This fact has been verified with model-based acoustic inversion processors[2], when comparing e.g. inverted temperature profiles with those measured by a conductivity-temperature-depth profiler (CTD). Naturally, if it was possible to convert oceanographic forecasts into 'equivalent' water column parameters, and real geometric/geoacoustic properties into 'equivalent' counterparts, then in principle, the predicted acoustic signal error would vanish. This observation has been made in the past, by comparison of acoustic cost functions facing real acoustic data with synthetic data from either oceanographic forecasts, measures or 'equivalents' [15]. Also, in [3], the value of acoustic inversion has been demonstrated in finding the acoustic model parameters (also because of a lack of environmental knowledge)-or 'equivalent' parameters-and their uncertainty, to make acoustically optimized sonar performance predictions for the present time.

This paper presents an acoustic predictor formulated as a Bayesian estimator, which takes into account the environmental model 'equivalence' in predicting the acoustic signal, and eventual oceanographic errors/biases. The acoustic signal to predict is modeled as the realization of a random variable, function of a random environment. The departing information consists of water column measures, oceanographic forecasts, acoustic data and geometric/geoacoustic properties. The posterior PDF of the acoustic signal conditioned on this information is estimated, allowing the direct definition of MMSE, MAP and median estimates, derived from acoustic error cost functions, as implied by the standard Bayesian framework. These cost functions oppose to environmental cost functions used in standard acoustic prediction-except as in $[10,12,18]$, considering interdisciplinary cost functions-, and are expected to give improved acoustic predictions, in average. This is because the environmental shifts needed for solving the acoustic modeling (forward) problem are 'learned' by solving an inverse problem. The method is supported by simulations run with water column measurements and forecasts obtained for the Maritime Rapid Environmental Assessment 2003 (MREA'03) sea trial[6].

Regarding the paper structure, sec. 2 describes the Bayesian acoustic predictor theoretical background, sec. 3 presents simulation results, and sec. 4 concludes and gives some perspectives. 


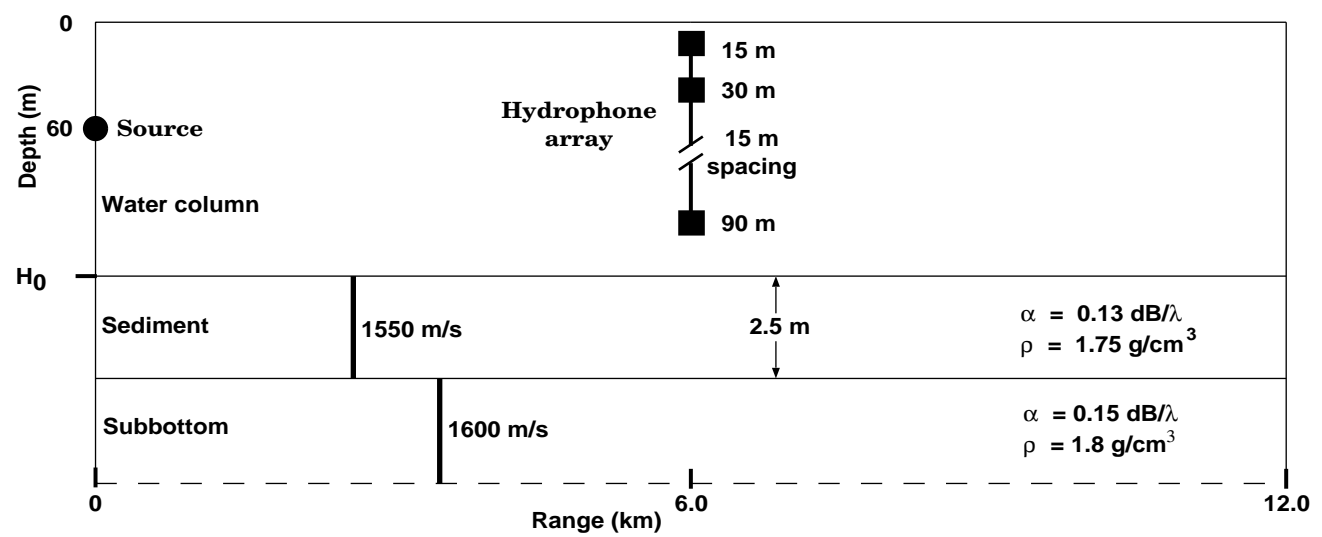

Fig. 1. Acoustic propagation transect: data inversion in $6 \mathrm{~km}$ range and acoustic prediction up to $12 \mathrm{~km}$ range. No mismatch scenario $H_{0}=123 \mathrm{~m}$ or mismatch $H_{0}=123.2 \mathrm{~m}$.

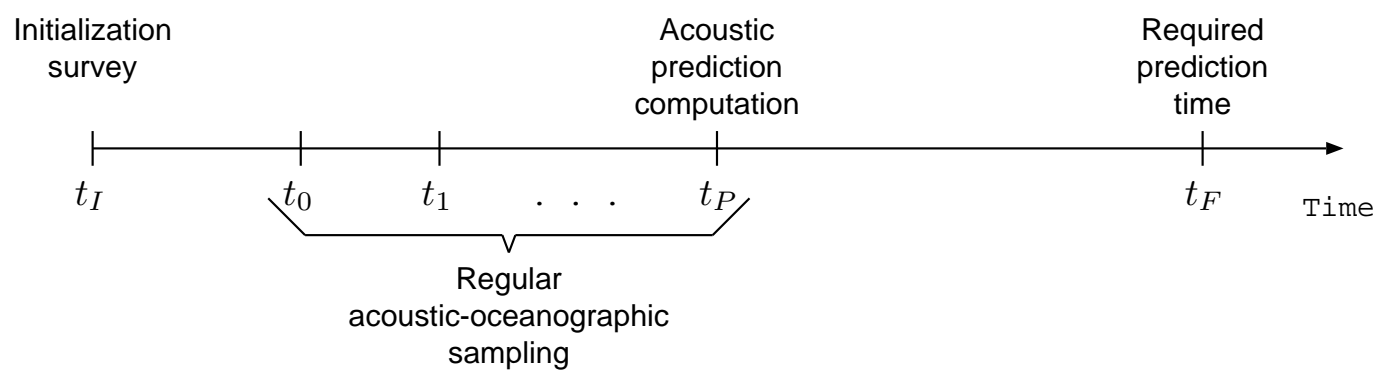

Fig. 2. Acoustic prediction time line. After the initialization of the oceanographic model at $t_{I}$, acoustic and oceanographic samples of the oceanic area of interest are taken on a regular basis, and the oceanographic model is run for the area, giving forecasts for $t_{0}, t_{1}, \ldots, t_{P}$ (present time) and $t_{F}$ (required acoustic prediction time). All the acoustic-oceanographic information is combined, to predict the acoustics at $t_{F}$.

\section{Bayesian acoustic prediction}

Let us consider the problem of predicting the acoustic field $\mathbf{u}(r, z, f)$ at range $r$, depth $z$ and frequency $f$ in the (two-dimensional) ocean transect represented in fig. 1 at a time $t_{F} \geq t_{P}$, where $t_{P}$ is the present time. The dependence on $(r, z, f)$ will sometimes be dropped for convenience. The environment in fig. 1 is a shallow water scenario with characteristics similar to real conditions observed in the MREA'03 sea trial[6], here modeled as a 3 layers-acoustic waveguide. Two components are essential to solve the problem at hand: an oceanographic model and an acoustic observation system. Here, the acoustic observation system is fixed, and composed of an acoustic source and a 6 hydrophonesarray-see fig. 1.

Figure 2 schematizes the underlying time line of the acoustic prediction process, described as follows. At a narrow time window centered on $t_{I}$, an ocean- 
ographic model is initialized and calibrated for the area of interest with extensive oceanographic/atmospheric measures. The model produces forecasts of the water column conditions at (or interpolated/extrapolated to) times $t_{0}, t_{1}, \ldots, t_{P}, t_{F}$. The forecasts of interest here may be either direct physical quantities such as temperature, salinity or sound speed, or compact representations of these quantities such as empirical orthogonal functions (EOFs) coefficients. For a compact notation, the forecasts for $t_{0}, t_{1}, \ldots, t_{P}$ are stacked in vector $\boldsymbol{\omega}$, and the one for $t_{F}$ is in vector $\mathbf{o}_{F}$. At time $t_{0}$, space-time-dense regular oceanographic and acoustic observations start taking place.

During a narrow time window centered on each time $t_{k}, k=0, \ldots, P$, the acoustic observation process produces a set of acoustic data snapshots stacked in vector $\mathbf{a}_{k}$, where each snapshot contains the acoustic signals received on the hydrophones -see fig. 1 . The corresponding water column conditions $\mathbf{w}_{k}$ are assumed time-invariant during the observation window. The data sets $\mathbf{a}_{k}$ and $\mathbf{w}_{k}$ are stacked into vectors $\boldsymbol{\alpha}$ and $\boldsymbol{\psi}$, respectively. Some of the bottom properties are range-independent in the transect and known to the user, forming vector $\mathbf{g}_{k}$. Every acoustic data set $\mathbf{a}_{k}$ is inverted for the environmental properties, by means of standard acoustic inversion techniques (see for example [19]). To simulate common acoustic modeling mismatch issues as stated in the introduction, it is assumed that the user considers an environmental model with an erroneous water depth, by assuming a value of $H_{0}=123.2 \mathrm{~m}$. A water depth mismatch of only $20 \mathrm{~cm}$ is ridiculously small in practical terms where errors are generally of the order of $5 \%$ of the water depth. The reasons of this choice will be made clear in sec. 3 . The mismatched environmental model is considered for both acoustic inversion and prediction. The 'equivalent' environment is described by the 'water column' and 'geometric/geoacoustic' vectors $\boldsymbol{\epsilon}_{w}$ and $\mathbf{e}_{b}$, respectively. Vector $\boldsymbol{\epsilon}_{w}$ contains all the time-varying components $\mathbf{e}_{w k}$ at $t_{k}, k=0,1, \ldots, F$, and vector $\mathbf{e}_{b}$ contains the 'equivalent' bottom parameters. Table 1 lists important vectors in the notation.

Prior to sea trials, it is a common practice to sample the environment-by means of CTDs, thermistor chains, sediment cores, echo-boomers, etc. This information thus obtained can be combined with information from historical databases or previous inversions, and synthesized in an empirical prior PDF of the environment. Also, the acoustic inversion outcome assumes often the form of a posterior environmental PDF, as will be seen later. These facts motivate a stochastic representation of the environment, which implies a stochastic model also for the signal component of the acoustic signals. This framework led naturally to a Bayesian formulation of the estimation problem, where $\mathbf{u}$ is the random variable realization to estimate.

The first step in deriving the estimate $\hat{\mathbf{u}}(r, z, f)$ of the acoustic field is the determination of the posterior PDF of $\mathbf{u}, p\left(\mathbf{u} \mid \boldsymbol{\omega}, \mathbf{o}_{F}, \mathbf{g}_{k}, \boldsymbol{\alpha}, \boldsymbol{\psi}\right)$, conditioned on all the available data. By applying standard probability relations, and taking 
Table 1

Selected vectors employed in the notation.

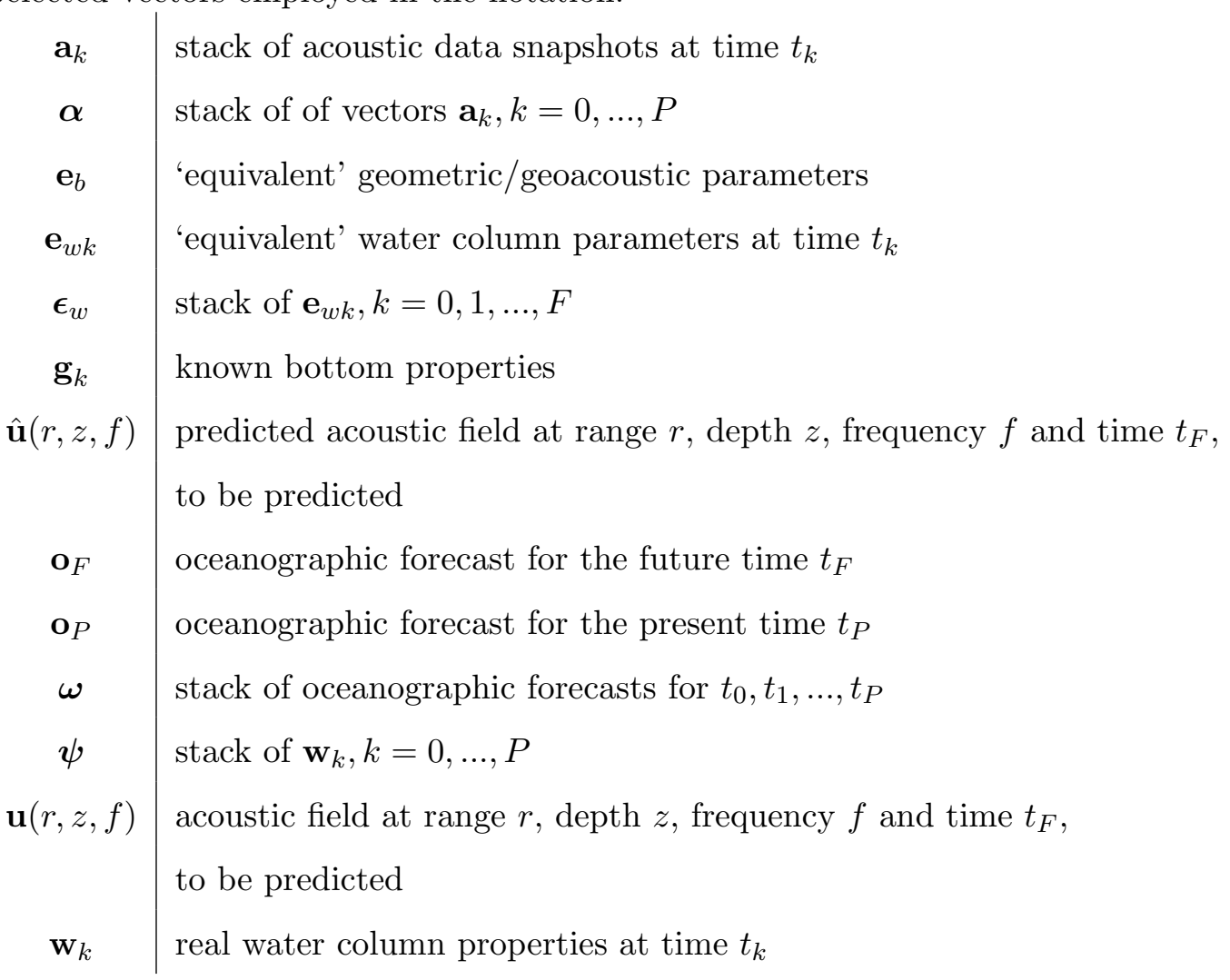

into account that the environmental realizations $\mathbf{e}_{w F}$ and $\mathbf{e}_{b}$ contain the whole information about $\mathbf{u}$,

$$
\begin{aligned}
& p\left(\mathbf{u} \mid \boldsymbol{\omega}, \mathbf{o}_{F}, \mathbf{g}_{k}, \boldsymbol{\alpha}, \boldsymbol{\psi}\right) \\
& \quad=\iint p\left(\mathbf{u} \mid \mathbf{e}_{w F}, \mathbf{e}_{b}, \boldsymbol{\omega}, \mathbf{o}_{F}, \mathbf{g}_{k}, \boldsymbol{\alpha}, \boldsymbol{\psi}\right) p\left(\mathbf{e}_{w F}, \mathbf{e}_{b} \mid \boldsymbol{\omega}, \mathbf{o}_{F}, \mathbf{g}_{k}, \boldsymbol{\alpha}, \boldsymbol{\psi}\right) \mathrm{d} \mathbf{e}_{b} \mathrm{~d} \mathbf{e}_{w F} \\
& \quad=\iint p\left(\mathbf{u} \mid \mathbf{e}_{w F}, \mathbf{e}_{b}\right) p\left(\mathbf{e}_{w F}, \mathbf{e}_{b} \mid \boldsymbol{\omega}, \mathbf{o}_{F}, \mathbf{g}_{k}, \boldsymbol{\alpha}, \boldsymbol{\psi}\right) \mathrm{d} \mathbf{e}_{b} \mathrm{~d} \mathbf{e}_{w F} .
\end{aligned}
$$

Since the required acoustic signal $\mathbf{u}$ can be generated deterministically with the realizations $\mathbf{e}_{w F}$ and $\mathbf{e}_{b}$ by resorting to the acoustic propagation model at hand, and by writing the environment-to-acoustics transformation as $\mathbf{u}=$ $U\left(\mathbf{e}_{w F}, \mathbf{e}_{b}\right),(1)$ simplifies to

$$
\begin{aligned}
& p\left(\mathbf{u} \mid \boldsymbol{\omega}, \mathbf{o}_{F}, \mathbf{g}_{k}, \boldsymbol{\alpha}, \boldsymbol{\psi}\right) \\
& \quad=\iint \delta\left(\mathbf{u}-U\left(\mathbf{e}_{w F}, \mathbf{e}_{b}\right)\right) p\left(\mathbf{e}_{w F}, \mathbf{e}_{b} \mid \boldsymbol{\omega}, \mathbf{o}_{F}, \mathbf{g}_{k}, \boldsymbol{\alpha}, \boldsymbol{\psi}\right) \mathrm{d} \mathbf{e}_{b} \mathrm{~d} \mathbf{e}_{w F},
\end{aligned}
$$

with $\delta$ the Dirac distribution. The right-hand PDF in (2) can be written as

$$
p\left(\mathbf{e}_{w F}, \mathbf{e}_{b} \mid \boldsymbol{\omega}, \mathbf{o}_{F}, \mathbf{g}_{k}, \boldsymbol{\alpha}, \boldsymbol{\psi}\right)=\frac{p\left(\mathbf{e}_{w F}, \mathbf{e}_{b}, \boldsymbol{\omega}, \mathbf{o}_{F}, \mathbf{g}_{k}, \boldsymbol{\alpha}, \boldsymbol{\psi}\right)}{\iint p\left(\mathbf{e}_{w F}, \mathbf{e}_{b}, \boldsymbol{\omega}, \mathbf{o}_{F}, \mathbf{g}_{k}, \boldsymbol{\alpha}, \boldsymbol{\psi}\right) \mathrm{d} \mathbf{e}_{b} \mathrm{~d} \mathbf{e}_{w F}}
$$


Assuming that the water column properties (measured, forecast and 'equivalent') are statistically independent from the bottom properties, and taking into account that the oceanographic forecasts add no information to that contained in $\boldsymbol{\psi}$ and $\mathbf{e}_{w F}$ about $\boldsymbol{\alpha}$, the PDF in the numerator of (3) is equal to

$$
\begin{aligned}
& p\left(\mathbf{e}_{w F}, \mathbf{e}_{b}, \boldsymbol{\omega}, \mathbf{o}_{F}, \mathbf{g}_{k}, \boldsymbol{\alpha}, \boldsymbol{\psi}\right) \\
& \quad=p\left(\mathbf{e}_{w F}, \boldsymbol{\omega}, \mathbf{o}_{F}, \boldsymbol{\psi}\right) p\left(\mathbf{e}_{b}, \mathbf{g}_{k}\right) p\left(\boldsymbol{\alpha} \mid \mathbf{e}_{w F}, \boldsymbol{\omega}, \mathbf{o}_{F}, \boldsymbol{\psi}, \mathbf{e}_{b}, \mathbf{g}_{k}\right) \\
& \quad=p\left(\boldsymbol{\omega}, \mathbf{o}_{F}, \boldsymbol{\psi}\right) p\left(\mathbf{e}_{w F} \mid \boldsymbol{\omega}, \mathbf{o}_{F}, \boldsymbol{\psi}\right) p\left(\mathbf{g}_{k}\right) p\left(\mathbf{e}_{b} \mid \mathbf{g}_{k}\right) p\left(\boldsymbol{\alpha} \mid \mathbf{e}_{w F}, \boldsymbol{\psi}, \mathbf{e}_{b}, \mathbf{g}_{k}\right) .
\end{aligned}
$$

Taking into account that, apart from acoustic noise, the variables $\boldsymbol{\epsilon}_{w}$ and $\mathbf{e}_{b}$ contain the whole information about $\boldsymbol{\alpha}$, the right-hand PDF in (4) is equal to

$$
\begin{aligned}
p\left(\boldsymbol{\alpha} \mid \mathbf{e}_{w F}, \boldsymbol{\psi}, \mathbf{e}_{b}, \mathbf{g}_{k}\right) & =\int p\left(\boldsymbol{\alpha} \mid \boldsymbol{\epsilon}_{w}, \mathbf{e}_{w F}, \boldsymbol{\psi}, \mathbf{e}_{b}, \mathbf{g}_{k}\right) p\left(\boldsymbol{\epsilon}_{w} \mid \mathbf{e}_{w F}, \boldsymbol{\psi}, \mathbf{e}_{b}, \mathbf{g}_{k}\right) \mathrm{d} \boldsymbol{\epsilon}_{w} \\
& =\frac{p(\boldsymbol{\alpha})}{p\left(\mathbf{e}_{b}\right)} \int \frac{p\left(\boldsymbol{\epsilon}_{w}, \mathbf{e}_{b} \mid \boldsymbol{\alpha}\right) p\left(\boldsymbol{\epsilon}_{w} \mid \mathbf{e}_{w F}, \boldsymbol{\psi}\right)}{p\left(\boldsymbol{\epsilon}_{w}\right)} \mathrm{d} \boldsymbol{\epsilon}_{w} .
\end{aligned}
$$

By replacing (5) in (4), it follows that

$$
\begin{aligned}
p\left(\mathbf{e}_{w F}, \mathbf{e}_{b}, \boldsymbol{\omega}, \mathbf{o}_{F}, \mathbf{g}_{k}, \boldsymbol{\alpha}, \boldsymbol{\psi}\right)=\frac{p\left(\boldsymbol{\omega}, \mathbf{o}_{F}, \boldsymbol{\psi}\right) p\left(\mathbf{e}_{w F} \mid \boldsymbol{\omega}, \mathbf{o}_{F}, \boldsymbol{\psi}\right) p\left(\mathbf{g}_{k}\right) p\left(\mathbf{e}_{b} \mid \mathbf{g}_{k}\right) p(\boldsymbol{\alpha})}{p\left(\mathbf{e}_{b}\right)} \\
\times \int \frac{p\left(\boldsymbol{\epsilon}_{w}, \mathbf{e}_{b} \mid \boldsymbol{\alpha}\right) p\left(\boldsymbol{\epsilon}_{w} \mid \mathbf{e}_{w F}, \boldsymbol{\psi}\right)}{p\left(\boldsymbol{\epsilon}_{w}\right)} \mathrm{d} \boldsymbol{\epsilon}_{w} .
\end{aligned}
$$

This allows to express (3) as

$$
\begin{aligned}
p\left(\mathbf{e}_{w F}, \mathbf{e}_{b} \mid \boldsymbol{\omega}, \mathbf{o}_{F}, \mathbf{g}_{k}, \boldsymbol{\alpha}, \boldsymbol{\psi}\right) & \\
= & \frac{p\left(\mathbf{e}_{w F} \mid \boldsymbol{\omega}, \mathbf{o}_{F}, \boldsymbol{\psi}\right) p\left(\mathbf{e}_{b} \mid \mathbf{g}_{k}\right)}{n\left(\boldsymbol{\omega}, \mathbf{o}_{F}, \boldsymbol{\psi}, \mathbf{g}_{k}, \boldsymbol{\alpha}\right) p\left(\mathbf{e}_{b}\right)} \int \frac{p\left(\boldsymbol{\epsilon}_{w}, \mathbf{e}_{b} \mid \boldsymbol{\alpha}\right) p\left(\boldsymbol{\epsilon}_{w} \mid \mathbf{e}_{w F}, \boldsymbol{\psi}\right)}{p\left(\boldsymbol{\epsilon}_{w}\right)} \mathrm{d} \boldsymbol{\epsilon}_{w},
\end{aligned}
$$

where the normalization term $n\left(\boldsymbol{\omega}, \mathbf{o}_{F}, \boldsymbol{\psi}, \mathbf{g}_{k}, \boldsymbol{\alpha}\right)$ simply assures an unitary integral for $p\left(\mathbf{e}_{w F}, \mathbf{e}_{b} \mid \boldsymbol{\omega}, \mathbf{o}_{F}, \mathbf{g}_{k}, \boldsymbol{\alpha}, \boldsymbol{\psi}\right)$. Finally, the required posterior PDF in (1) can be rewritten as

$$
\begin{aligned}
p\left(\mathbf{u} \mid \boldsymbol{\omega}, \mathbf{o}_{F}, \mathbf{g}_{k}, \boldsymbol{\alpha}, \boldsymbol{\psi}\right) & \\
=\frac{1}{n\left(\boldsymbol{\omega}, \mathbf{o}_{F}, \boldsymbol{\psi}, \mathbf{g}_{k}, \boldsymbol{\alpha}\right)} \iint & \frac{\delta\left(\mathbf{u}-U\left(\mathbf{e}_{w F}, \mathbf{e}_{b}\right)\right) p\left(\mathbf{e}_{w F} \mid \boldsymbol{\omega}, \mathbf{o}_{F}, \boldsymbol{\psi}\right) p\left(\mathbf{e}_{b} \mid \mathbf{g}_{k}\right)}{p\left(\mathbf{e}_{b}\right)} \\
& \times \int \frac{p\left(\boldsymbol{\epsilon}_{w}, \mathbf{e}_{b} \mid \boldsymbol{\alpha}\right) p\left(\boldsymbol{\epsilon}_{w} \mid \mathbf{e}_{w F}, \boldsymbol{\psi}\right)}{p\left(\boldsymbol{\epsilon}_{w}\right)} \mathrm{d} \boldsymbol{\epsilon}_{w} \mathrm{~d} \mathbf{e}_{b} \mathrm{~d} \mathbf{e}_{w F}
\end{aligned}
$$

The determination of $p\left(\mathbf{u} \mid \boldsymbol{\omega}, \mathbf{o}_{F}, \mathbf{g}_{k}, \boldsymbol{\alpha}, \boldsymbol{\psi}\right)$ is seen as the crucial step in the acoustic prediction process. With the information carried by the posterior PDF, three non-linear Bayesian acoustic predictors are then derived[7]: 


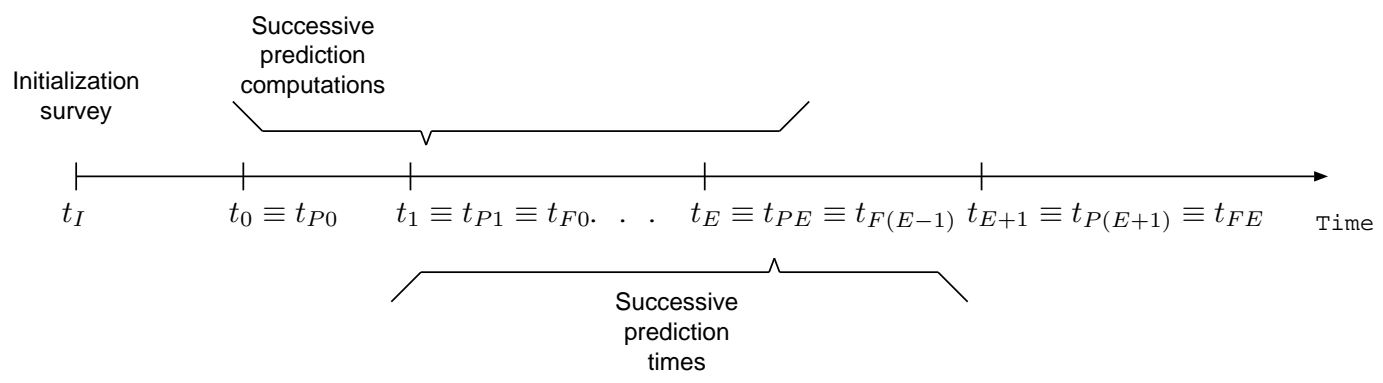

Fig. 3. Acoustic prediction simulation time line. At each present time $t_{P i}$, the acoustic field at $t_{F i} \equiv t_{P(i+1)}$ is predicted. The prediction window $t_{F i}-t_{P i}$ is $23 \mathrm{~min}$.

$$
\begin{aligned}
\hat{\mathbf{u}}_{M M S E}(r, z, f) & =\int \mathbf{u} p\left(\mathbf{u} \mid \boldsymbol{\omega}, \mathbf{o}_{F}, \mathbf{g}_{k}, \boldsymbol{\alpha}, \boldsymbol{\psi}\right) \mathrm{d} \mathbf{u} \\
\hat{\mathbf{u}}_{M A P}(r, z, f) & =\arg \max _{\mathbf{u}} p\left(\mathbf{u} \mid \boldsymbol{\omega}, \mathbf{o}_{F}, \mathbf{g}_{k}, \boldsymbol{\alpha}, \boldsymbol{\psi}\right) \\
\hat{\mathbf{u}}_{M E D}(r, z, f) & =\text { median of } p\left(\mathbf{u} \mid \boldsymbol{\omega}, \mathbf{o}_{F}, \mathbf{g}_{k}, \boldsymbol{\alpha}, \boldsymbol{\psi}\right)
\end{aligned}
$$

\section{Simulations}

The prediction of the acoustic field $\mathbf{u}(r, z, f)$ is illustrated in the following, using synthetic noiseless acoustic data generated with collected CTD data and oceanographic forecasts produced for the MREA'03 sea trial[6] by the Navy Coastal Ocean Model[14]. The considered acoustic model was the normalmode model SNAP[4].

Due to the importance of acoustic inversion in determining the 'equivalent' environmental model, as stated in the introduction, the acoustic system depicted in fig. 1 takes observations between Julian day (JD) 162 (June 12 ${ }^{\text {th }}$ ) and 175 (June $25^{\text {th }}$ ). The emitted signal is a sum of $N_{\text {freq }}=10$ equally spaced tones from 710 to $800 \mathrm{~Hz}$. The observations are performed in a regular time grid as shown in fig. 3. The aim of the acoustic predictor is to estimate the acoustic field in the transect shown in fig. 1 . At each (running) time $t_{P i}$, the data acquired till $t_{P i}$ is considered, to predict the acoustic field for the next time sample $t_{F i}=t_{P i}+23 \mathrm{~min}$. The acoustic prediction takes place separately in each scenario without/with environmental mismatch depicted in fig. 1 . The water depth mismatch is the only source of model 'equivalence'.

It was chosen to represent the water column sound speed profiles (SSPs) as linear combinations of a depth-dependent functional basis formed by the first 2 (dimensionless) EOFs drawn from the CTD SSP data sequentially acquired in a large $142 \times 87.9 \mathrm{~km}$ area around the Elba Island, in the period May $28^{\text {th }}-$ June $25^{\text {th }}$, as shown in fig. 4 . The EOFs are shown in fig. 5 , and account for $87.2 \%$ 


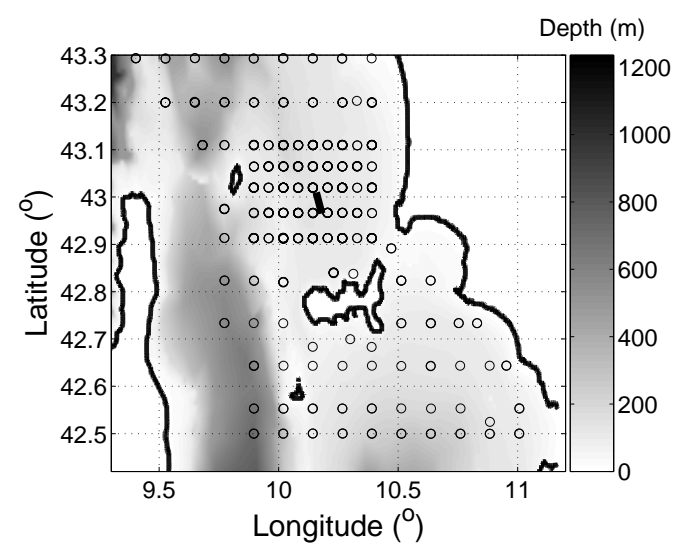

Fig. 4. Location of the CTD casts in the MREA'03 sea trial (circles) and bathymetric-range-independent transect considered in the simulations, centered on $\approx$ $\left(10.2^{\circ}, 43^{\circ}\right)$ (longitude,latitude).

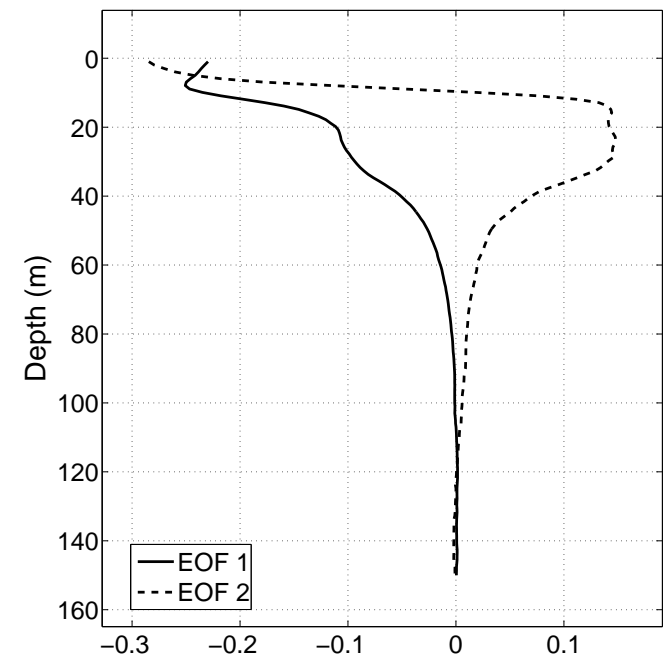

Fig. 5. Empirical orthogonal functions of the measured sound speed profiles during the MREA'03 sea trial.

of the SSP variance. The oceanographic forecasts were space-time linearly interpolated to the CTD casts, and then, both real and predicted SSPs were linearly interpolated to the regular observation time samples. Fig. 6 shows the difference between the projection of the measured and the forecast profiles onto the first EOF. There is an equal trend between the measured and predicted EOF coefficient. The prediction error decreases in average after JD 164. However, it is always highly non-stationary, with an estimated mean of 18.2 ${ }^{\circ} \mathrm{C}$, thus indicating also a bias of the oceanographic forecasts, in terms of the first EOF coefficient. This is not surprising, since NCOM restricts in situ data to remote sensed SSH and SST, assimilating this data and atmospheric data to a dynamic model, and considering historical relationships between water surface and column properties-being a model suitable for real-time operation.

In a performance comparison guideline, two acoustic predictors are applied. 


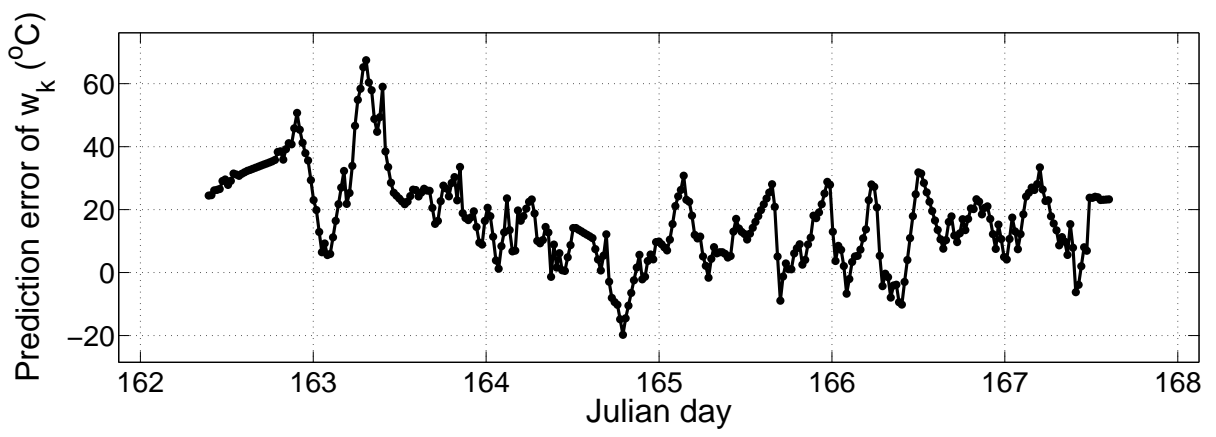

Fig. 6. Difference between the projection onto the first empirical orthogonal function, of the measured and NCOM predicted sound speed profiles.

The first is stated as the standard predictor. It feeds the acoustic model with the oceanographic forecast of the first EOF coefficient, $\mathbf{o}_{F}$. The second predictor assumes the form of the Bayesian estimators in (8), feeding the model with the 'equivalent' coefficient. Here, as a preliminary study of the developed acoustic predictor, the acoustic data was inverted for a single parameter $\mathbf{e}_{w P}$, the first EOF coefficient, using the depth-coherent, frequency-incoherent Bartlett processor

$$
P\left(\mathbf{e}_{w P i}\right)=1-\sum_{k=1}^{N_{f r e q}} \tilde{\mathbf{w}}^{\mathrm{H}}\left(\mathbf{e}_{w P i}\right) \hat{\mathbf{R}}_{i} \tilde{\mathbf{w}}\left(\mathbf{e}_{w P i}\right)
$$

where $\tilde{\mathbf{w}}$ is an unitary norm acoustic field candidate along the hydrophone array, and $\hat{\mathbf{R}}_{i}$ is an estimate of the correlation matrix of $\mathbf{a}_{i}$. During the acoustic inversion, the search bounds for $\mathbf{e}_{w P}$ were $-32.4{ }^{\circ} \mathrm{C}$ and $57.8{ }^{\circ} \mathrm{C}$, and the discretization in the search space was $0.354^{\circ} \mathrm{C}$.

Since the acoustic data was inverted for only one environmental parameter, there is only one degree of freedom to guarantee environmental model 'equivalence'. Thus, to guarantee acoustic field a large similarity between the acoustic field generated with the 'equivalent' environment and that generated with the true environment, it was necessary to allow a water depth mismatch of $<20$ $\mathrm{cm}$. Obviously, in future studies, where more environmental parameters are allowed to vary, the environmental mismatches will assume realistic values.

In the scenario with no environmental mismatch, it is expected that the equivalent parameter $\mathbf{e}_{w P i}$ coincides with the real $\mathbf{w}_{P i}$. Both in acoustic inversion and prediction, all the environmental parameters apart from the first EOF coefficient are fixed to the assumed known values in fig. 1. The data flow in the two predictors is sketched in fig. 7 . 
(a)

(b)

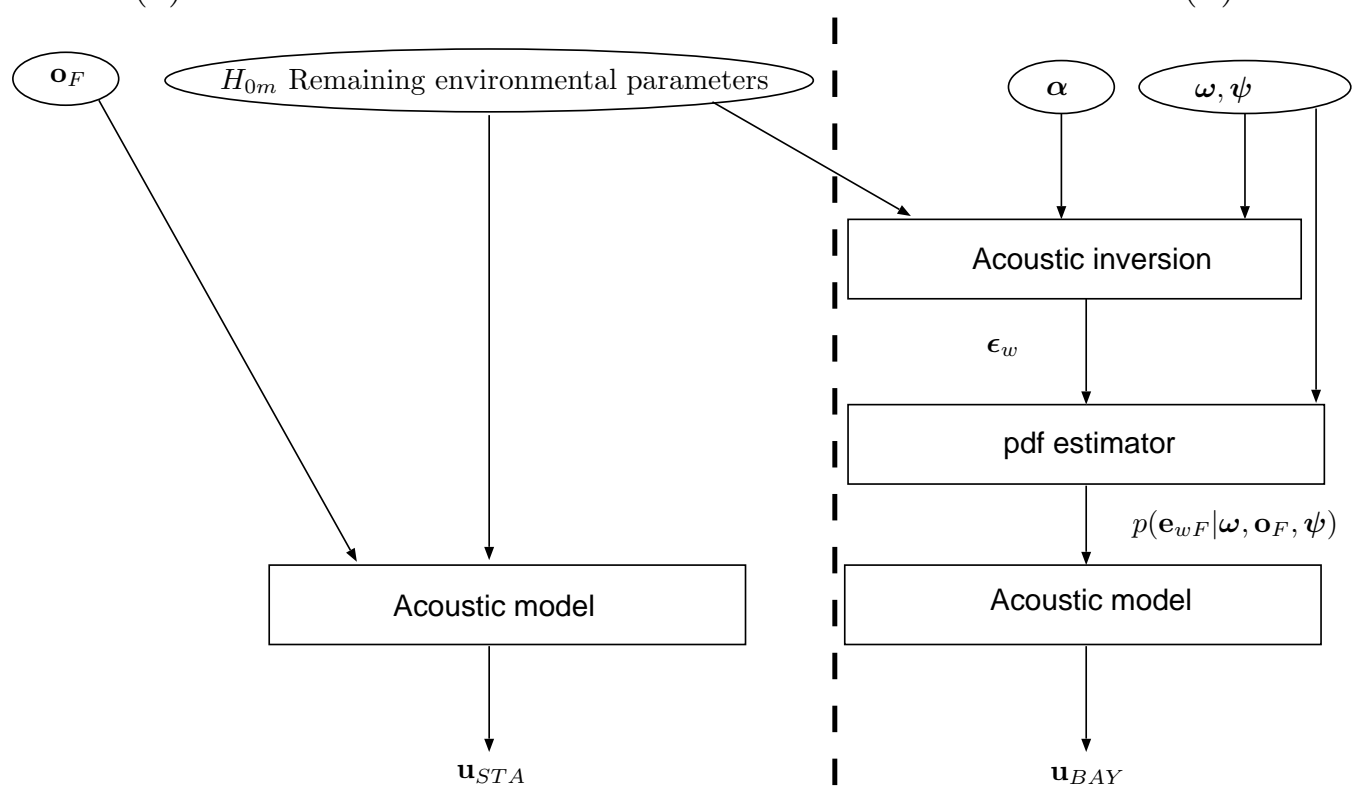

Fig. 7. Data flow in the standard (a) and Bayesian (b) predictors.

\subsection{Practical issues}

Since the only environmental parameter inverted for is the first EOF coefficient, the proposed Bayesian approach simplifies to

$$
\begin{aligned}
p\left(\mathbf{u} \mid \boldsymbol{\omega}, \mathbf{o}_{F}, \boldsymbol{\alpha}, \boldsymbol{\psi}\right) & \\
=\frac{1}{n\left(\boldsymbol{\omega}, \mathbf{o}_{F}, \boldsymbol{\psi}, \boldsymbol{\alpha}\right)} \int \delta & \left(\mathbf{u}-U\left(\mathbf{e}_{w F}\right)\right) p\left(\mathbf{e}_{w F} \mid \boldsymbol{\omega}, \mathbf{o}_{F}, \boldsymbol{\psi}\right) \\
& \times \int \frac{p\left(\boldsymbol{\epsilon}_{w} \mid \boldsymbol{\alpha}\right) p\left(\boldsymbol{\epsilon}_{w} \mid \mathbf{e}_{w F}, \boldsymbol{\psi}\right)}{p\left(\boldsymbol{\epsilon}_{w}\right)} \mathrm{d} \boldsymbol{\epsilon}_{w} \mathrm{~d} \mathbf{e}_{w F} .
\end{aligned}
$$

Considering that the future 'equivalent' coefficient $\mathbf{e}_{w F}$ may not add a significant information about the past and present 'equivalent' coefficients in $\boldsymbol{\epsilon}_{w}$, relatively to the real coefficients in $\boldsymbol{\psi}$, one may approximate $p\left(\boldsymbol{\epsilon}_{w} \mid \mathbf{e}_{w F}, \boldsymbol{\psi}\right) \approx$ $p\left(\boldsymbol{\epsilon}_{w} \mid \boldsymbol{\psi}\right)$, and (10) becomes

$$
p\left(\mathbf{u} \mid \boldsymbol{\omega}, \mathbf{o}_{F}, \boldsymbol{\alpha}, \boldsymbol{\psi}\right)=N\left(\boldsymbol{\omega}, \mathbf{o}_{F}, \boldsymbol{\psi}, \boldsymbol{\alpha}\right) \int \delta\left(\mathbf{u}-U\left(\mathbf{e}_{w F}\right)\right) p\left(\mathbf{e}_{w F} \mid \boldsymbol{\omega}, \mathbf{o}_{F}, \boldsymbol{\psi}\right) \mathrm{d} \mathbf{e}_{w F},
$$

where $N\left(\boldsymbol{\omega}, \mathbf{o}_{F}, \boldsymbol{\psi}, \boldsymbol{\alpha}\right)$ is a normalizing term. Summarily, as stated by (11), it is required to estimate the future 'environment' $\mathbf{e}_{w F}$, whose information is contained in the posterior $\operatorname{PDF} p\left(\mathbf{e}_{w F} \mid \boldsymbol{\omega}, \mathbf{o}_{F}, \boldsymbol{\psi}\right)$.

Taking into account the possible non-stationarity of the oceanographic field, it may be argued that old data has little information on future quantities. Thus, 
it is reasonable to consider that the oceanographic forecasts and real water column conditions in past times $t_{P j}, j<i$, add no significant information about the 'equivalent' $\mathbf{e}_{w F}$, w.r.t. the present and future quantities $\left(\mathbf{o}_{P}, \mathbf{w}_{P}\right)$ and $\mathbf{o}_{F}$, respectively. With this reasoning, the density $p\left(\mathbf{e}_{w F} \mid \boldsymbol{\omega}, \mathbf{o}_{F}, \boldsymbol{\psi}\right)$ can be approximated as

$$
\begin{aligned}
p\left(\mathbf{e}_{w F} \mid \boldsymbol{\omega}, \mathbf{o}_{F}, \boldsymbol{\psi}\right) & \approx p\left(\mathbf{e}_{w F} \mid \mathbf{o}_{P}, \mathbf{o}_{F}, \mathbf{w}_{P}\right) \\
& =\frac{p\left(\mathbf{e}_{w F}, \mathbf{o}_{P}, \mathbf{o}_{F}, \mathbf{w}_{P}\right)}{p\left(\mathbf{o}_{P}, \mathbf{o}_{F}, \mathbf{w}_{P}\right)} .
\end{aligned}
$$

The estimation of (12) reduces to the estimation of the numerator, since the denominator is a constant PDF value. If it was to compute a 3D joint density of the variables $\mathbf{o}_{P}, \mathbf{o}_{F}$ and $\mathbf{w}_{P}$, the estimation would be straightforward, and done by binning the increasingly gathered real and predicted water column profiles, whose relative difference in time is $t_{F}-t_{P}$, into a histogram. The variable $\mathbf{e}_{w F}$ requires special attention, since there are no scalar outcomes available at each time $t_{P i}$. Instead, as a product of acoustic inversion, which is typically an ill-posed problem, the 'natural' information about $\mathbf{e}_{w P i}$ is contained in $p\left(\mathbf{e}_{w P i} \mid \mathbf{a}_{i}\right)$. In fact, it might also be reasonable to consider scalar Bayesian estimates of $\mathbf{e}_{w P i}$ derived from $p\left(\mathbf{e}_{w P i} \mid \mathbf{a}_{i}\right)$ as outcomes to fill the required 4D histogram. Nevertheless, this could cause problems, in the case of high acoustic inversion ambiguity, in which two or more concurrent maxima of the cost function could lead to alternate disparate values of the environmental estimate. So, here, the $4 \mathrm{D}$ histogram, at each bin for $\left(\mathbf{o}_{P}, \mathbf{o}_{F}, \mathbf{w}_{P}\right)$, was filled with the a posteriori PDFs $p\left(\mathbf{e}_{w k} \mid \mathbf{a}_{k}\right)$. There is an underlying stationarity assumption for computing the empirical PDF, that stationarity, for the case at hand, being limited as shown in fig. 6. The available oceanographic data from measurements, forecasts and acoustic inversion is sparse in the required 4D space. This required interpolation/extrapolation of the empirical PDF bins already filled till $t_{P i}$. Once $p\left(\mathbf{e}_{w F} \mid \mathbf{o}_{P}, \mathbf{o}_{F}, \mathbf{w}_{P}\right)$ is estimated, the acoustic signal corresponding to each outcome of $\mathbf{e}_{w F}$ is computed by forward modeling. Afterwards, this ensemble of acoustic signals is binned, and each bin weighted according to $p\left(\mathbf{e}_{w F} \mid \boldsymbol{\omega}, \mathbf{o}_{F}, \boldsymbol{\psi}\right)$, to produce an histogram which is the estimate of $p\left(\mathbf{u} \mid \boldsymbol{\omega}, \mathbf{o}_{F}, \boldsymbol{\alpha}, \boldsymbol{\psi}\right)$. This means that the accuracy of the predicted acoustic field is rather dependent on the accuracy of $p\left(\mathbf{e}_{w F} \mid \boldsymbol{\omega}, \mathbf{o}_{F}, \boldsymbol{\psi}\right)$. At the end, the predicted acoustic field emerges as a trivial application of (8) to the obtained $p\left(\mathbf{u} \mid \boldsymbol{\omega}, \mathbf{o}_{F}, \boldsymbol{\alpha}, \boldsymbol{\psi}\right)$.

\subsection{Results}

This section presents results of acoustic prediction obtained with the Bayesian and the standard acoustic predictor. It starts by illustrating the steps of the 
(a)

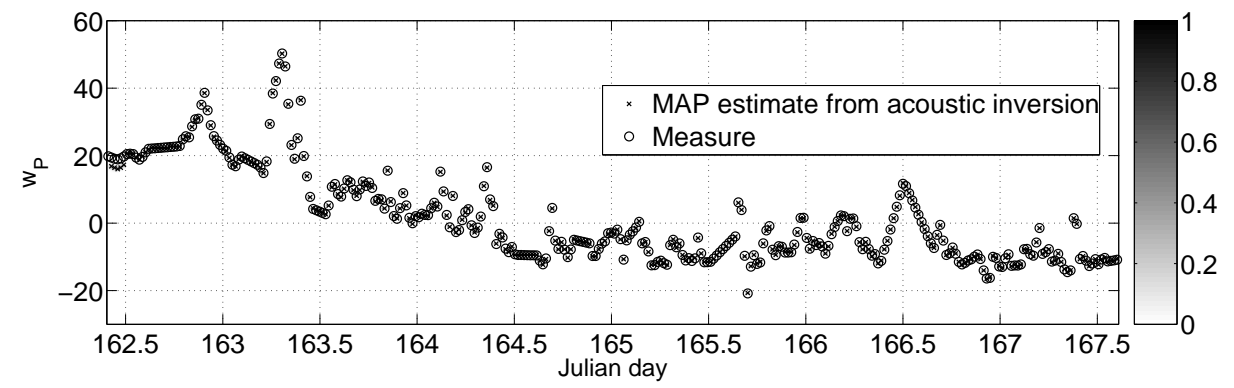

(b)

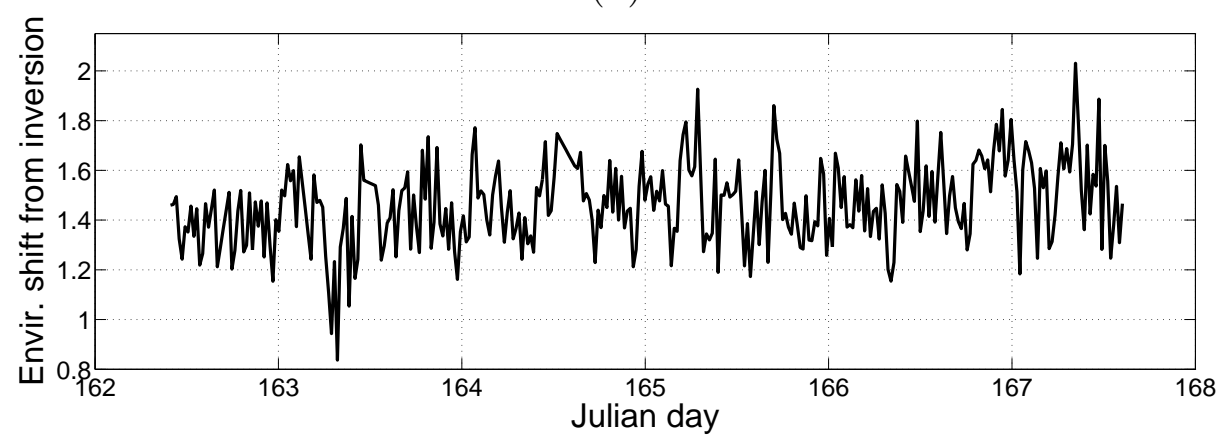

Fig. 8. Acoustic inversion results for the first EOF coefficient: measurements and inversion results without mismatch (a) and estimate error with $20 \mathrm{~cm}$ water depth mismatch (b).

Bayesian predictor, shown in fig. 7. In the first step, acoustic inversion, the obtained results are shown in fig. 8 (a) and (b), for the cases without/with environmental mismatch, respectively. Each column of fig. 8 (a) contains a posterior environmental function $p\left(\mathbf{e}_{w P i} \mid \mathbf{a}_{i}\right)$, which, for the noiseless acoustic data at hand, is very narrow and centered on the true environmental values, as expected. For the case with environmental mismatch in fig. 8 (b), the results are shown as the environmental estimation error by acoustic inversion. Naturally, the observed shifts, around $1.5{ }^{\circ} \mathrm{C}$, are caused by the mismatched water depth.

In the second step, PDF estimation, the obtained result for the last time sample of the acoustic prediction trial, JD 167.6, is shown in fig. 9. Each posterior environmental PDF estimate $\hat{p}\left(\mathbf{e}_{w F} \mid \mathbf{o}_{P}, \mathbf{o}_{F}, \boldsymbol{\psi}\right)$ was obtained from the interpolation/extrapolation of an empirical PDF, as explained in sec. 3.1. The posterior environmental PDF estimates are compared to the results of acoustic inversion, $\hat{p}\left(\mathbf{e}_{w F} \mid \mathbf{a}_{F}\right)$, carried for the same time sample. The latter PDF is taken as a reference, since shows which environmental values generate the future acoustic field most likely. The PDFs $\hat{p}\left(\mathbf{e}_{w F} \mid \mathbf{o}_{P}, \mathbf{o}_{F}, \boldsymbol{\psi}\right)$ are in good agreement with the PDFs $\hat{p}\left(\mathbf{e}_{w F} \mid \mathbf{a}_{F}\right)$. This was expected for this time sample, since the oceanographic prediction error attains stationarity previously to $t_{P(E+1)}$, as seen in fig. 6, implying that the results from interpolation/extrapolation of the empirical PDF do not deviate significantly from reality. The case of 
(a)

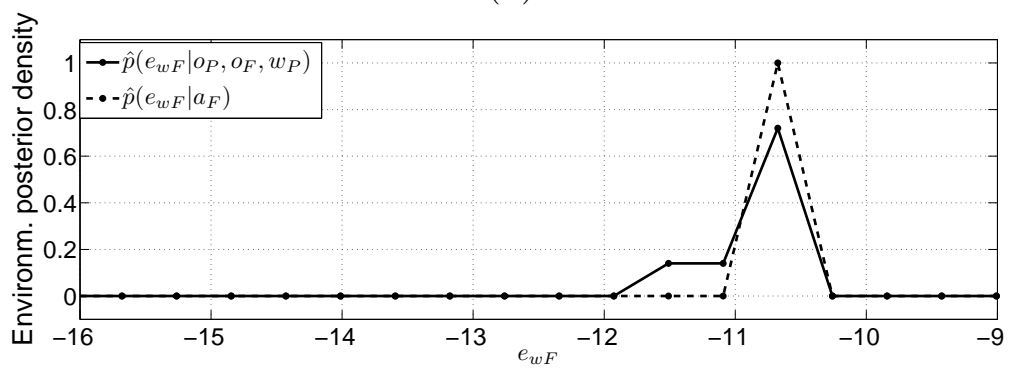

(b)

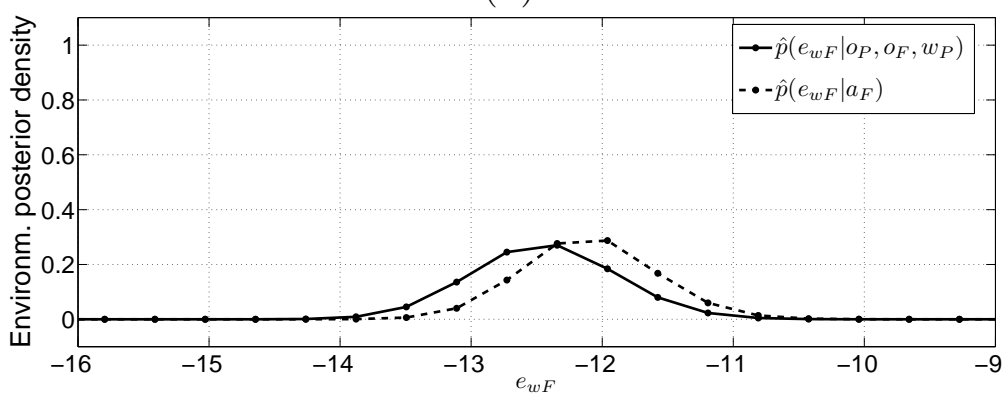

Fig. 9. Predictive posterior environmental PDFs $\hat{p}\left(\mathbf{e}_{w F} \mid \mathbf{o}_{P}, \mathbf{o}_{F}, \boldsymbol{\psi}\right)$, for the last prediction time sample, JD 167.6, in the cases considering the correct (a) or incorrect (b) water depth. These estimates of the future 'equivalent' EOF coefficient PDF are compared to PDFs $\hat{p}\left(\mathbf{e}_{w F} \mid \mathbf{a}_{F}\right)$ obtained from acoustic inversion.

considering the correct water depth in fig. 9 (a) does not differ in its essence and result from the case of the incorrect water depth in fig. 9 (b). In the case at hand, by considering the correct water depth, has given sharp PDFs from acoustic inversion, while the incorrect depth implied broader PDFs, which is understandable. In either case, the structure of the PDFs is registered in $\hat{p}\left(\mathbf{e}_{w F}, \mathbf{o}_{P}, \mathbf{o}_{F}, \boldsymbol{\psi}\right)$, with the samples gathered along time. Thus, as seen in fig. 9 (a), the PDF $\hat{p}\left(\mathbf{e}_{w F}, \mathbf{o}_{P}, \mathbf{o}_{F}, \boldsymbol{\psi}\right)$ has been trained to generate sharp functions $\hat{p}\left(\mathbf{e}_{w F} \mid \mathbf{o}_{P}, \mathbf{o}_{F}, \boldsymbol{\psi}\right)$ centered on the true environmental values-which for the last time sample is $\approx-10.7^{\circ} \mathrm{C}$ (see also fig. 8 (a))-, while in (b), generates broader functions deviated $\approx 1.5^{\circ} \mathrm{C}$ from reality (see also fig. $8(\mathrm{~b})$ ).

The predicted environment, obtained from the empirical posterior density interpolation/extrapolation, as mentioned in sec. 3.1, is in good agreement with the reference environment represented by $p\left(\mathbf{e}_{w P(E+1)} \mid \mathbf{a}_{P(E+1)}\right)$. This was expected for this sample, since the oceanographic prediction error attains stationarity previously to $t_{P(E+1)}$, as seen in fig. 6 .

The quality of the estimates of $p\left(\mathbf{e}_{w F} \mid \mathbf{o}_{P}, \mathbf{o}_{F}, \mathbf{w}_{P}\right)$ is illustrated along time in fig. 10. These predictive environmental posterior density estimates are rather a consequence of the oceanographic predictor stationarity. One can refer to fig. 6 and find a direct relation between the oceanographic prediction error stationarity and the error PDF error. For example, for $t_{F i}<163, t_{F i} \approx 164.5, t_{F i} \approx$ 166.9 and $t_{F i}>167.5$, the oceanographic prediction error exhibits smooth 


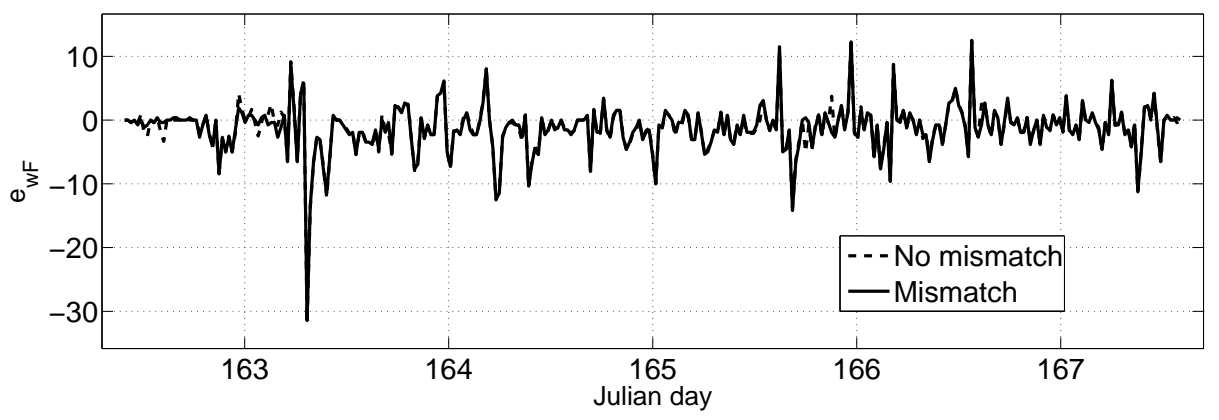

Fig. 10. Error of the estimates of the predictive environmental posterior $\hat{p}\left(\mathbf{e}_{w F} \mid \mathbf{o}_{P}, \mathbf{o}_{F}, \boldsymbol{\psi}\right)$ along time. The error is defined at each time as the difference between the values that maximize $\hat{p}\left(\mathbf{e}_{w F} \mid \mathbf{o}_{P}, \mathbf{o}_{F}, \boldsymbol{\psi}\right)$ and $p\left(\mathbf{e}_{w F} \mid \mathbf{a}_{F}\right)$, respectively.

variations, corresponding to weak errors in the posterior PDF. Conversely, periods of high oceanographic error variation, as $t_{F i} \approx 163.5, t_{F i}=164$ and $165.5<t_{F i}<166.5$ correspond to larger errors in the posterior PDF. This is explained by interpolation/extrapolation errors in $p\left(\mathbf{e}_{w F} \mid \mathbf{o}_{P}, \mathbf{o}_{F}, \mathbf{w}_{P}\right)$, misled by the unpredictable behavior of the particular oceanographic forecasts.

The acoustic field was generated for the $12 \mathrm{~km}$-transect in fig. 1, for the first and last frequencies of the emitted signal during the acoustic observation. At each time sample, a cost function indicating the quality of the acoustic estimate was defined as

$$
\phi=20 \log _{10} \sum_{r} \sum_{z} \sum_{f}\left|\mathbf{u}(r, z, f)-\mathbf{u}_{E S T}(r, z, f)\right|,
$$

where $\mathbf{u}(r, z, f)$ designates the true acoustic field and $\mathbf{u}_{E S T}(r, z, f)$ designates any of: the standard acoustic predictor $\hat{\mathbf{u}}_{S T A}(r, z, f)$ or the Bayesian predictors (8). The acoustic field estimated by the standard and Bayesian MMSE acoustic predictors, for JD 164.9, are compared in fig. 3.2. The MMSE acoustic predictor exhibits a smaller error than the standard predictor in the whole transect, at both frequencies. The error in (13) for all predictors along selected time samples is shown in fig. 12. For these time samples, the Bayesian predictors errors are always smaller than the standard predictor error, by 7 to 14 $\mathrm{dB}$, what proves the efficiency of including acoustic inversion in the acoustic prediction process.

\section{Conclusions and perspectives}

An acoustic predictor has been presented, which incorporates acoustic inversion as an environmental parameter tuner. The main contribution is the derivation of an estimator which optimizes for acoustic cost functions, instead 

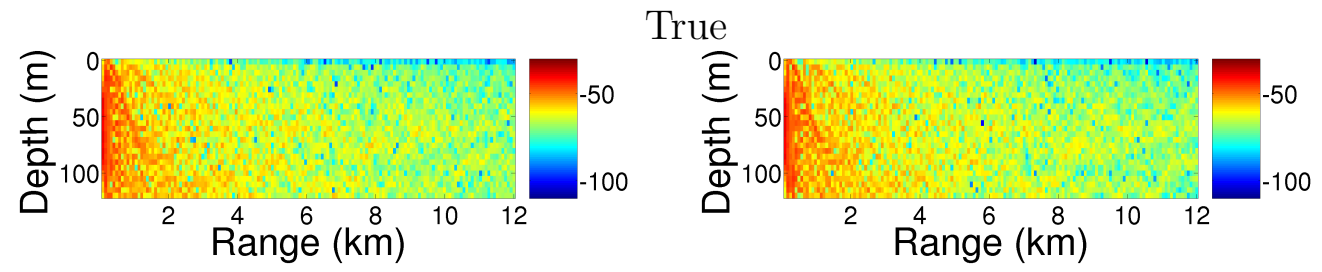

Standard: error
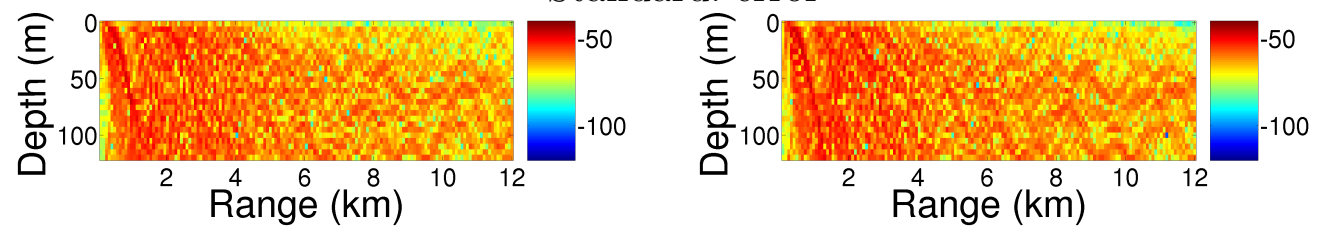

MMSE: error
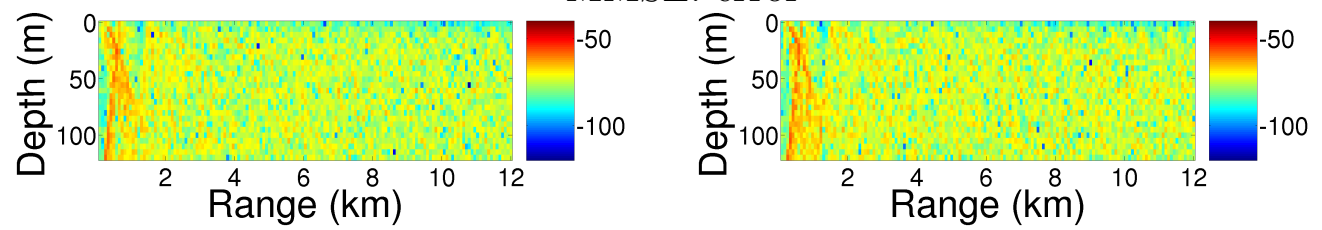

Fig. 11. True acoustic field and acoustic predictions errors obtained for JD 164.9, for the standard and MMSE predictors, at 710 and $800 \mathrm{~Hz}$. The true field is represented as $20 \log _{10}|\mathbf{u}(r, z, f)|$, while the error fields are represented as $20 \log _{10}\left|\mathbf{u}(r, z, f)-\mathbf{u}_{E S T}(r, z, f)\right|$.

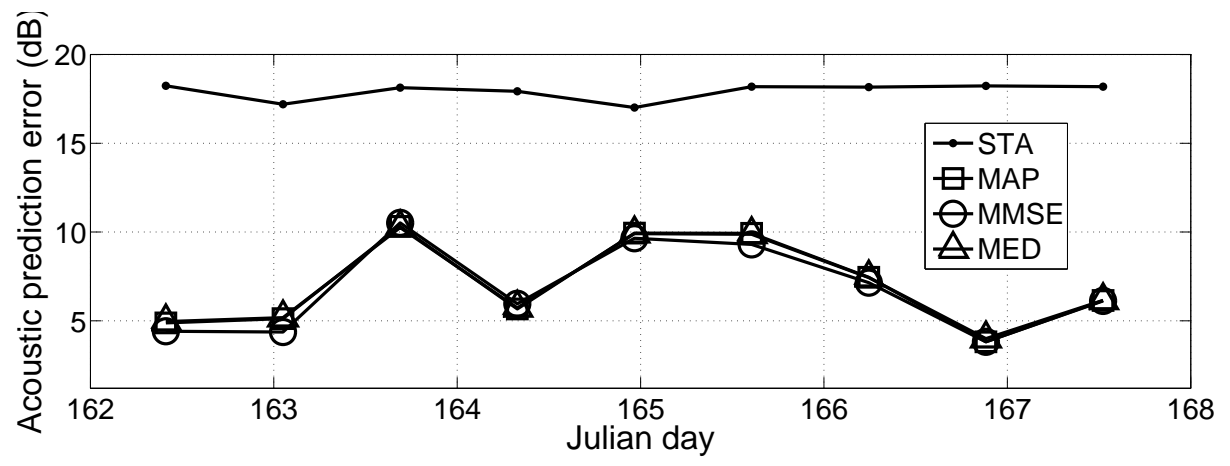

Fig. 12. Acoustic prediction error of the standard (STA) and Bayesian predictors.

of oceanographic cost functions, as in standard procedures.

The fact that the prediction of the acoustic field departs naturally from oceanographic predictions for the future time of interest, and from the application of an acoustic propagation model-parameterized by several environmental parameters whose information is not contained in oceanographic models- motivated the incorporation of a process of estimation of the the latter parameters, namely acoustic inversion. In this paper, a Bayesian framework has been described theoretically to arrive at the predicted acoustic field, assimilating information from different sources: oceanographic forecasts and measures, acoustic data and environmental parameters obtained from acoustic inversion. 
In the acoustic modeling component, an important issue is the fact that the environmental description is often incomplete, what can be represented by errors in some environmental parameters. Naturally, in order to obtain accurate acoustic outputs, these errors should be compensated with errors in other parameters. The resulting group of environmental parameters has been referred to as the 'acoustically equivalent environmental model', although a residual error of the acoustics generated with the 'equivalent model' is anticipated. Thus, for the objective of acoustic prediction, the importance of acoustic inversion is twofold, serving as an estimator of environmental parameters which may not be available to the end user, and as an estimator of the compensating environmental errors.

The proposed acoustic predictor was tested with a very simple yet illustrative scenario with simulated acoustic data with oceanographic measures and forecasts from the MREA'03 sea trial. The requirement was to predict the acoustic field in a transect containing an acoustic source and a vertical array. The oceanographic quantity of relevance was the coefficient of the first empirical orthogonal function (EOF) of the sound speed profiles gathered at sea. In the simulations, the cases of environmental mismatch/no mismatch were analyzed, where the environmental mismatch was reduced to an error in the water depth. In both acoustic inversion and prediction, only the coefficient of the first EOF was allowed to vary. The environmental mismatch is not a significant issue for acoustic prediction, provided the 'equivalent' parameters correspond to sufficient acoustic degrees of freedom, in order to compensate the environmental errors. This issue is being studied at the moment, by increasing the environmental mismatch and the number of 'equivalent' parameters, implying the full application of the theoretical expressions derived here.

The prediction of the future 'equivalent' environment has a significant impact in the acoustic predictor, and it was seen that its quality is dependent mainly on the oceanographic predictor. Better future environmental estimates are obtained with more stationary oceanographic predictors. This is a natural drawback of the environmental estimator being based on the interpolation/extrapolation of an empirical density function.

The acoustic field predictions illustrate the superiority of the proposed acoustic predictor relatively to a standard predictor, with improvements of 7 to $14 \mathrm{~dB}$, as an integral measure along range, depth and frequency. While the standard predictor feeds the acoustic model with the oceanographic forecast and the remaining environmental information, regardless of its possible mismatch, the proposed predictor uses acoustic inversion to tune the environment, tracking eventual environmental mismatches, in order to optimize the Bayesian acoustic cost functions implicit in the presented estimators.

Future developments will apply the Bayesian approach to cases in which water 
column measures are unavailable, to cases of moving acoustic observation systems, and to cases in which the oceanographic forecast can be fed directly to the acoustic model, and compensated by the geometric/geoacoustic parameters. Another interesting issue is whether a joint density of the acoustic field can give better estimates than the individual densities for each range-depthfrequency sample.

\section{Acknowledgements}

We thank the partial funding of Fundação para a Ciência e Tecnologia - FCT under POSI, POCTI and POCI programs, and scholarship no. SFRH/BD/9032/2002. Acknowledgements are addressed also to Emanuel Coelho, for conducing the MREA'03 sea trial, and to Peter Gerstoft, for prompt help and improvements of the SAGA inversion package.

\section{References}

[1] Elisseeff P., Schmidt H., Johnson M., Herold D., Chapman N.R., and McDonald M.M., "Acoustic tomography of a coastal front in Haro Strait, British Columbia", J. Acoust. Soc. Am., 106, No. 1, 169-184, 1999.

[2] P. Gerstoft and D.F. Gingras, "Parameter estimation using multi-frequency range-dependent acoustic data in shallow water", J. Acoust. Soc. America, 99, 2839-2850, 1996.

[3] Chen-Fen Huang, Peter Gerstoft, and William S. Hodgkiss, "Validation of statistical estimation of transmission loss in the presence of geoacoustic inversion uncertainty", J. Acoust. Soc. America, 120, 1932-1941, 2006.

[4] F.B. Jensen and M.C. Ferla, "SNAP: The SACLANTCEN normal-mode acoustic propagation model," SM-121, SACLANT Undersea Research Centre, La Spezia, Italy (1979).

[5] F.B. Jensen, W.A. Kuperman, M.B. Porter and H. Schmidt, "Computational Ocean Acoustics", American Institute of Physics, 1993.

[6] S.M. Jesus, C. Soares and A.J. Silva, Acoustic Oceanographic Buoy testing during the Maritime Rapid Environmental Assessment 2003 sea trial, SiPLAB, University of Algarve, Report, 04/03, Faro, 2003.

[7] Steven M. Kay, Fundamentals of statistical signal processing: estimation theory, 1993, ISBN 0-13-345711-7, Prentice-Hall, Inc., Upper Saddle River, NJ, USA.

[8] Lermusiaux, P.F.J., C.-S. Chiu and A.R. Robinson, "Modeling Uncertainties in the Prediction of the Acoustic Wavefield in a Shelfbreak Environment", 
Proceedings of the 5th ICTCA, May 21-25, 2001, in Theoretical and Computational Acoustics 2001, E.-C. Shang, Q. Li and T.F. Gao (Eds.), World Scientific Publishing Co., 191-200.

[9] P.F.J. Lermusiaux, P.J. Robinson and W.G. Leslie, "Advanced interdisciplinary data assimilation: Filtering and smoothing via Error Subspace Statistical Estimation", Proceedings of "The OCEANS 2002 MTS/IEEE" conference, IEEE, Holland Publications, 795-802, 2002.

[10] P.F.J. Lermusiaux and C.-S. Chiu, 417-424, Four-dimensional data assimilation for coupled physical-acoustical fields, Proceedings of the "Acoustic Variability, 2002" conference in Lerici, Italy, 2002.

[11] Lermusiaux P.F.J., C.-S. Chiu, G.G. Gawarkiewicz, P. Abbot, A.R. Robinson, R.N. Miller, P.J. Haley, W.G. Leslie, S.J. Majumdar, A. Pang and F. Lekien, 2006. Quantifying Uncertainities in Ocean Predictions. (.pdf). Oceanography, Special issue on "Advances in Computational Oceanography", T. Paluszkiewicz and S. Harper, Eds., Vol. 19, 1, 92-105.

[12] Lermusiaux P.F.J., 2006. Uncertainty Estimation and Prediction for Interdisciplinary Ocean Dynamics (.pdf). J. Computational Physics, Special issue on "Uncertainty Quantification", J. Glimm and G. Karniadakis, Eds., In press.

[13] C.J. Lozano, A.R. Robinson, H.G. Arango, A. Gangopadhyay, N.Q. Sloan, P.J. Haley and W.G. Leslie, Elsevier Science, An interdisciplinary ocean prediction system: Assimilation strategies and structured data models, Modern Approaches to Data Assimilation in Ocean Modelling, P. Malanotte-Rizzoli, Ed., Elsevier Oceanography Series, Elsevier Science, 413452, 1996.

[14] Martin P., "Description of the Navy Coastal Ocean Model version 1.0", report NRL/FR/7322-00-9962, Naval Research Laboratory (2000).

[15] N. Martins, C. Soares and S. Jesus, Environmental and acoustic assessment: The AOB concept, Journal of Marine Systems, Volume 69, Issues 1-2, , Maritime Rapid Environmental Assessment - New Trends in Operational Oceanography, January 2008, Pages 114-125.

[16] A.R. Robinson and D. Lee, Introduction: Ocean Variability, Acoustic Propagation and Coupled Models, 1-6, AIP Press, AIP Series in Modern Acoustics and Signal Processing, 1994.

[17] A.R. Robinson, P. Abbot, P.F.J. Lermusiaux and L. Dillman, Transfer of uncertainties through physical-acoustical-sonar end-to-end systems: a conceptual basis, Acoustic Variability, 2002, N.G. Pace and F.B. Jensen (Eds.), SACLANTCEN, 603-610, 2002.

[18] Robinson A. R., and Lermusiaux P. F. J., "Prediction systems with data assimilation for coupled ocean science and ocean acoustics", in: Sixth International Conference on Theoretical and Computational Acoustics (ICTCA), Honolulu, HI, 11 August, 2003, pp. 325-342. 
[19] A. Tolstoy. Matched Field Processing for Underwater Acoustics. World Scientific, Singapore, 1993. 